\title{
La incorporación de la educación en Derechos Humanos en el sistema de educación púbica, sector primaria, en el Uruguay ${ }^{1}$
}

Incorporation of Human Rights education in the public education system, primary sector, in Uruguay

\author{
Ana Laura Piñeyro Jardim ${ }^{2}$ \\ Universidad de la República - Uruguay
}

Revista Derechos en Acción ISSN 2525-1678/ e-ISSN 2525-1686

Año 5/Nº 15, Otoño 2020 (21 marzo a 21 junio), 299-317

DOl: https://doi.org/10.24215/25251678e400

\section{Introducción}

Desde 2008 el Uruguay cuenta con una nueva Ley General de Educación ( $\mathrm{N}^{\mathrm{o}}$ 18.437- 2008) y, a partir del mes de marzo de 2009, con un nuevo programa único de Educación Pública de nivel inicial y primaria, de aplicación en todo el territorio nacional. En estos dos documentos se observa la explícita enunciación, tanto de los principios y objetivos, como de los contenidos de la EDH. En la nueva Ley de Educación, en su artículo 40, se determina la creación de diferentes líneas transversales que deberán implementarse en todas las modalidades del Sistema Nacional de Educación (artículo 20), entre las que

\footnotetext{
Trabajo realizando en base a la Tesis de Maestría en Derecho Humanos realizada en la Facultad de Ciencias Jurídicas y Sociales de la Universidad Nacional de La Plata.

2 Licenciada en Psicología (Universidad de la Republica-UDELAR, Uruguay). Magister en Derechos Humanos (Universidad Nacional de La Plata-UNLP, Argentina)
} 
se encuentra (artículo 40.1) ${ }^{3}$ la educación en derechos humanos $(\mathrm{EDH})^{4}$. Esta está considerada como un derecho en sí mismo y un componente del derecho a la educación.

A su vez en la fundamentación del actual programa de educación inicial y primaria, se encuentran los derechos humanos como el marco ${ }^{5}$, tanto en la dimensión del conocimiento como en la práctica social integral.

Este nuevo marco, de incorporación explícita de la EDH tanto en la Ley General de Educación como en el Programa vigente de Educación Inicial y Primaria, implica cambios en la concepción del ámbito educativo. Sin embargo, la mera enunciación de estos conceptos en los documentos no cambia automáticamente la práctica y concepciones que orientan el quehacer cotidiano de docentes, familias y estudiantes. Se trata de un proceso complejo, que involucra ámbitos sociales, culturales, jurídicos, administrativos, vivencias, experiencias y condiciones materiales que permitan llevar adelante el trabajo. Consciente de la densidad de fenómenos que el hecho educativo encierra, la investigación de tesis que se presenta pretendió indagar, la forma en que estos cambios de paradigma se procesan en el sistema educativo de primaria.

3 Ley N 18.437 Artículo. 40,1: La educación en derechos humanos tendrá como propósito que los educandos, sirviéndose de conocimientos básicos de los cuerpos normativos, desarrollen las actitudes e incorporen los principios referidos a los derechos humanos fundamentales. Se considerará la educación en derechos humanos como un derecho en sí misma, un componente inseparable del derecho a la educación y una condición necesaria para el ejercicio de todos los derechos humanos."

4 La otras líneas transversales que se encuentran en el art. 40 de la Ley N 18.437: b) la educación ambiental para el desarrollo humano sostenible; c) la educación artística; d) la educación científica; e) la educación lingüística; f) la educación a través del trabajo; g) la educación para la salud; h) la educación sexual; i) la educación física, la recreación y el deporte.

5 Programa de Educación Inicial y Primaria. Publicado por CODICEN-CEP. Montevideo, Uruguay, marzo 2009 (P. 12) "Las ideas directrices, que en el contexto del Programa dan sentido a la educación y se orientan hacia la construcción del ciudadano del siglo XXI son:

- Democracia Social que se fundamenta en los Derechos Humanos, la diversidad, la participación, el posicionamiento ético, el trabajo digno y la solidaridad.

- Integralidad a través de la estética, la educación ambiental, la promoción de la salud y la sexualidad. 
Para ello se analizá:

(1) los aspectos que se incorporan sobre la EDH en los documentos normativos de la educación pública en el Uruguay y su incidencia en las prácticas educativas, para lo cual se compara la actual ley general de educación con las anteriores;

(2) el nuevo currículo para identificar los elementos incorporados y las metodologías sugeridas, y

(3) las prácticas educativas, en particular si estas se llevan adelante desde una perspectiva de EDH y cómo.

Esta investigación aspira a ser un insumo de evaluación de la política pública educativa que se está llevando a cabo actualmente en el Uruguay. Pretende indagar la EDH desde una concepción de disciplina compleja ${ }^{6}$ e identificar fortalezas o debilidades en su planteo como eje transversal del Sistema Nacional de Educación Pública, en especial en primaria. Se confía en que permitirá comprender las vinculaciones entre la ley, el currículo y las prácticas educativas - los tres elementos que hacen a la incorporación de los derechos humanos en la educación- para poder establecer si se están articulando de manera coherente y eficiente, de manera total o parcial.

\section{Metodología}

Para esta tesis de Maestría en Derechos Humanos, se realizó un trabajo de investigación que tuvo diferentes fases:

En una primera instancia, tuvo lugar la construcción de un marco teórico, junto a una investigación documental centrada en la normativa educativa del Uruguay y su desarrollo histórico en relación a los diferentes períodos socio-políticos.

Se realizó la construcción de un marco de referencia que permite la explicitación de algunos conceptos en torno a los

\footnotetext{
6 El término "Complejo o Complejidad". Se refiere a una comprensión del mundo como un todo, compuesto por partes que se encuentran entrelazadas y, en esa interacción, generan nuevas características. Uno de sus pensadores más destacados en el ámbito educativo y de las ciencias humanas es Edgar Morin. (MORIN, 1998)
} 
cuales se llevó adelante todo el proceso de investigación. Es así que se elaboran una serie de recorridos teóricos, que permiten ir dando contenidos y encuadres de comprensión que acompañan el análisis realizado. Allí se referencia y define qué se entiende por derechos humanos, y una visión en la órbita del Derecho Internacional de los derechos humanos, del derecho a la Educación y a la EDH.

En el segundo momento de la investigación se realizaron una serie de entrevistas en profundidad a expertos en $\mathrm{EDH}$ que trabajan en áreas destacadas del sistema educativo y también a autoridades de la educación pública uruguaya que han sido protagonistas de la redacción de la normativa analizada. El objetivo fue indagar y comprender desde qué concepción y cómo ha sido la incorporación de la EDH en el sistema educativo uruguayo. ${ }^{7}$

En una última etapa se busco investigar las prácticas educativas en el terreno. Se tramitó el permiso, en el ámbito correspondiente y a través de las inspectoras generales del departamento de Montevideo, fueron designadas tres escuelas. ${ }^{8}$ El trabajo de campo consistió en una aproximación a las prácticas educativas con un enfoque etnográfico, a través de observación participante y entrevistas y talleres, con modalidad de Investigación Cualitativa.

La metodología de investigación cualitativa con orientación etnográfica, tiene raíces en la tradición de las ciencias sociales y supone una serie de condicionantes teóricas y metodológicas, donde se cruzan temas y disciplinas, en este caso la investigación en educación y la antropología. Esto lleva a que la metodología aplicada tenga características propias que se manifiestan en la

\footnotetext{
7 En motivos de extensión no se va hacer referencias explicitas a las entrevistas a expertos y autoridades.

8 El Consejo de Educación Inicial y Primaria (CEIP) de la Administración Nacional de Educación Pública (ANEP) divide a Montevideo en tres grandes zonas (oeste, centro y este) para ordenar la jurisdicción a la que corresponden las escuelas otorgando una inspección para cada región.
} 
modalidad de construcción del conocimiento: el modo como se llevan adelante las observaciones, la forma de análisis y elaboración de los conceptos y nociones teórico-metodológicas; y los procedimientos para obtener la confiabilidad y la validez. Esto implica que la búsqueda de conocimiento está orientada a la comprensión de ideas, de sentimientos y de significados, presentes en las descripciones obtenidas de las palabras y/o de las conductas y prácticas observadas (Tarrés, 2001).

\section{Marco teórico de referencia. La EDH propuesta con anclaje en el Derechos Internacional de los Derechos Humanos}

En el Derecho Internacional de los Derechos Humanos, en la órbita del Sistema Universal (ONU) como en el ámbito Interamericano (OEA), existe una coincidencia de criterios con respecto al derecho a la educación y a la EDH. Así como en el artículo 26.2 de la Declaración Universal de Derechos Humanos, en el "Protocolo Adicional a la Convención Americana sobre derechos humanos en materia de derechos económicos, sociales y culturales", el "Protocolo de San Salvador" en su artículo 13.2, se explicita la finalidad que debe tener la educación.

“... la educación deberá orientarse hacia el pleno desarrollo de la personalidad humana y del sentido de su dignidad y deberá fortalecer el respeto por los derechos humanos, el pluralismo ideológico, las libertades fundamentales, la justicia y la paz. ... la educación debe capacitar a todas las personas para participar efectivamente en una sociedad democrática y pluralista, lograr una subsistencia digna, favorecer la comprensión, la tolerancia y la amistad entre todas las naciones y todos los grupo raciales, étnicos o religiosos y promover las actividades a favor del mantenimiento de la paz."

9 Protocolo adicional a la Convención Americana sobre derechos humanos en materia de derechos económicos, sociales y culturales. (OEA, 1989) PROTOCOLO DE SAN SALVADOR. Art. 13,2. 
Entendida así, el recibir una educación sistemática, de calidad, que permita a todas y cada una de las personas comprender los derechos humanos desde la dimensión de respeto mutuo para una convivencia responsable con la dignidad propia y la de las otras personas, grupos y pueblos implica asumir a la EDH como un derecho humano en sí mismo.

Esta concepción ubica al sistema de educación formal y en especial a la etapa de educación primaria, en un lugar de importancia para la promoción, prevención y realización de los derechos humanos. Ya que la obligación de los Estados es generar una política pública educativa con acceso universal a todos los niños y niñas en edad escolar ${ }^{10}$, se hace relevante esta prestación de enseñanza con un enfoque de $\mathrm{EDH}$.

En este marco, las Naciones Unidas a través de la UNESCO y el ACDHUN ${ }^{11}$ vienen impulsando diferentes iniciativas, como ser: el Decenio para la Educación de los derechos humanos (1995-2005); un "Plan de Acción, Programa Mundial para la educación en derechos humanos" (que comenzó en 2005, y se encuentra actualmente en su cuarta etapa); y se proclamó en el 2011 la "Declaración sobre educación y formación en materia de derechos humanos".

"La meta de la educación en derechos humanos es forjar sociedades donde no se atropelle la dignidad humana. No basta con denunciar o castigar los abusos después que han ocurrido. Hay que evitarlo y para ello, es necesario que las personas conozcan sus derechos, los defiendan y, a la vez, respeten y defiendan su ejercicio por parte de los demás. La educación en derechos humano es un medio para promover ese conocimiento y respeto y, de esta manera, se convierte en un instrumento concreto de prevención de violaciones a los derechos humanos.

\footnotetext{
10 Articulo 13 y 14 del Pacto Internacional de Derechos Económicos, Sociales y Culturales, ONU, 1966.

11 Las siglas corresponden a : Organización de las Naciones Unidas para la Educación, la Ciencia y la Cultura, UNESCO y el Alto Comisionado para los Derechos Humanos ACDHUN
} 
Al mismo tiempo, se propone construir una convivencia pacífica y democrática, cada día más respetuosa de la vida y las libertades individuales, más igualitaria, justa y solidaria para todos y cada uno. En breve: más humana. Vista de esta manera es el motor de transformaciones individuales y sociales." (Rodino, AM. 1999: 4)

La EDH, considerada como un derecho en sí mismo, concepto que parte de las esferas internacionales. Llega al ámbito nacional con el desafío de poder transmitir los principios fundamentales de los derechos humanos, como ser la igualdad y la no discriminación, así como de profundizar la relación entre todos los derechos, destacando su interdependencia, indivisibilidad y universalidad.

Desde la normativa y estándares internacionales sobre derechos humanos, se deja claro la perspectiva integral y transversal que debe asumir la educación como derecho humano. Marca así una postura de protección y promoción de respeto hacia todas las personas para desarrollar sus potencialidades, transformar sus realidades de vida y construir una convivencia entre todos y todas, con pautas de respeto a la dignidad humana y a las diferentes culturas.

Los principios, objetivos de los derechos humanos se deberían encarnar en la experiencia cotidiana de las realidades vividas por las personas (Pérez Aguirre, 1999), desde sus propios contextos socio-culturales, y así fomentar y fortalecer sociedades donde se respete la dignidad humana y sus múltiples manifestaciones

Es en la órbita del Derecho Internacional de los Derechos Humanos, que podemos identificar la obligación de los Estados en adecuar su normativa interna, y llevar adelante políticas públicas donde la EDH esté presente, como estrategia para promover "las actitudes y el comportamiento necesario para que se respeten los derechos humanos de todos los miembros de la sociedad". ${ }^{12}$

12 Naciones Unidad, Plan de Acción, Programa Mundial para la EDH, primera etapa (Resolución 59/113B -Asamblea General) 
Es así que la EDH cobra fuerza como un derecho en sí misma con un doble sentido, conteniendo una concepción del ser humano y de una forma de relacionarse entre las personas, y como prácticas concretas de convivencia respetuosa (Pérez Aguirrez, 1999). Esto quiere decir que la EDH implica una acción que incluye o contiene la finalidad de que se educa en y para los derechos humanos (Magendzo, 2008). Es decir educar en la filosofía de los derechos humanos y también para generar prácticas sociales y educativas donde se respete la dignidad de todas las personas involucradas (Rodino, 1999, 2003).

...estamos mucho más allá del tema de la mera información y la 'instrucción' sobre los derechos humanos. Quizás sea más accesible, aunque nunca fácil, trasmitir información sobre ellos, su historia, el contenido de la Declaración Universal, las Convenciones... Pero educar es otra cosa. Educar es modificar las actitudes y las conductas. Es afectar los corazones, los estilos de vida, las convicciones. Y es evidente que esto no puede hacerse sino en el sentido de las actitudes del propio educador. No podemos concebir el proceso educativo más que como una especie de empatía, de mímesis de actitudes entre ambos sujetos del proceso educativo. $\mathrm{Y}$ esto me lleva a sospechar que la educación siempre será una tarea de carácter ético o no será. (Pérez Aguirre, L.1999)

\section{Marco normativo en Uruguay: Ley General de Educación $\mathbf{N}^{\mathbf{0}} \mathbf{1 8 . 4 3 7}$}

\section{Antecedentes en las leyes}

Se presenta una breve reseña sobre las leyes de educación que existieron en el Uruguay antes de la actual Ley General de Educación No 18.437, promulgada en diciembre de 2008.

Entre los antecedentes cercanos de la actual ley se encuentra la Ley de Educación $\mathrm{N}^{\circ} 14.101$ del 4 de enero de 1973, sancionada previo a la dictadura cívico-militar que afrontará el país a partir de junio de 1973 hasta marzo de 1985. Esta 
ley estableció un cambio en la estructura del sistema educativo que significó una organización más centralizada, con pérdida de autonomía para los diferentes niveles educativo (primaria, secundaria y enseñanza técnica).

En la restitución democrática se promulga el 28 de marzo de 1985 la "Ley de emergencia" No 15.739. En ella podemos encontrar referencias claras al momento social e histórico que estaba viviendo el país y se restaura un orden de funcionamiento y de actuación en consonancia con la democracia recientemente alcanzada.

En julio del 1990 se promulga la Ley $N^{\circ} 16.115$, que está compuesta de un artículo único, el cual establece la forma de organización de las autoridades de la Administración Nacional de Educación Pública (ANEP).

\section{Elementos de EDH en la Ley $N^{o} 18.437$}

La Ley $\mathrm{N}^{0} 18.437$ o Ley General de Educación regula toda la educación en el país incluyendo la primera infancia, los niveles de educación inicial, primario, secundario y terciario, educación policial, militar y educación no formal. Desde su inicio en el artículo $1^{13}$, ubica al tema de la educación como derecho humano fundamental y hace referencia a la obligación del Estado de garantizar dicho derecho para todos los habitantes a lo largo de la vida.

En el artículo 2 reconoce a la educación como bien público y social, con un alcance formativo de desarrollo integral de la persona humana, incorporando explícitamente el principio de no discriminación. La orientación que le da esta Ley a la educación (artículo 3) responde a un concepto integral de la vida humana bio-psico-socio-ambiental, incluyendo "el ejercicio responsable

\footnotetext{
13 Artículo 1: Declárese de interés general la promoción del goce y el efectivo ejercicio del derecho a la educación, como un derecho humano fundamental. El Estado garantizará y promoverá una educación de calidad para todos los habitantes, a lo largo de toda la vida, facilitando la continuidad educativa.
} 
de la ciudadanía, como factor esencial del desarrollo sostenible, la tolerancia, la plena vigencia de los derechos humanos, la paz y la comprensión entre los pueblos y las naciones."

El artículo 4 complementa al artículo 1, dando el marco de los derechos humanos para el ejercicio del derecho a la educación enmarcado en la Declaración Universal de Derechos Humanos, así como otros instrumentos internacionales ratificados por el país y la Constitución Nacional.

El artículo 5 identifica a las y los educandos como sujetos del derecho a la educación.

El capítulo II de la ley enmarca los principios de la educación desde la universalidad y la obligatoriedad (artículos 6 y 7). También son principios de la educación la participación, la libertad de enseñanza y la libertad de cátedra (artículos 9, 10 y 11). Adicionalmente, el artículo 8 destaca los principios de diversidad e inclusión educativa.

Y como ya se mencionó la Ley instaura ejes transversales, siendo el primero la EDH (articulo 40,1) la que se considera como un derecho en sí mismo y un componente del derecho a la educación. El artículo 110 se crea una Comisión asesora en EDH para el Sistema Nacional de Educación Pública, que involucra desde el nivel inicial hasta superior e universitario.

La Ley General de Educación en su artículo 48 plantea como un principio la participación de los diferentes actores de la comunidad educativa y de la sociedad en su conjunto. Como ámbito del ejercicio de la participación de sus diferentes referentes, la ley crea los Consejos de Participación en cada uno de los centros educativos de todo el país (artículos 76, 77 y 78). Estos deben estar integrados por estudiantes, educadores, madres o padres y representantes de la comunidad. Cada Consejo de Participación reglamentará la forma de elección de sus miembros, así como su funcionamiento. Se destaca que para los centros de educación Media Básica y Media Superior como los de educación Técnico Profesional, los Consejos de Participación deberán tener al menos un tercio de representantes estudiantiles. 


\section{Breve reflexión del Programa de Educación Inicial y Primaria}

En la actualización del presente Programa se observa que existe un progreso del Estado uruguayo en cuanto al tratamiento que se le da a la inserción y desarrollo de los derechos humanos dentro los programas curriculares de educación formal. Esto se ve tanto en su concepción más integral así como en el planteo de las áreas de conocimientos. En la justificación general del Programa se pueden comprender y aprehender con mayor cabalidad y complejidad los principios teórico-conceptuales de universalidad, integralidad y multidimensionalidad de los derechos humanos. A su vez se observa una mayor explicitación y tratamiento directo de temas de derechos humanos como contenido dentro del currículo escolar.

En el Programa se propone un tratamiento más destacado de temas y problemáticas vinculados con los derechos económicos, sociales y culturales, así como sobre la igualdad y no discriminación en diferentes esferas: de género, racial, social, laboral, económica y generacional. También se pretende generar reflexión y pensamiento crítico frente a situaciones de conflictividad social. Existe un trato importante y destacado de la educación para la ciudadanía y la democracia.

Asimismo está explicitada la búsqueda a través de los planteos didácticos de un sujeto de la educación más activo en su proceso de enseñanza aprendizaje, más protagonista. El posicionamiento y la reflexión frente a diferentes situaciones sociales son una metodología destacada desde el planteo teórico que realiza el programa. Esta búsqueda implica el desarrollo de una didáctica y una metodología que apunten a favorecer e impulsar habilidades y capacidades en los y las estudiantes que les permitan asumir una actitud de sujetos de derecho activos en la sociedad y tomar decisiones responsables y coherentes con los principios éticos de los derechos humanos.

En contraposición a los avances planteados en este nuevo Programa, se observa con preocupación un reducido tratamiento 
de conocimientos sobre los sistemas y los instrumentos internacionales de protección de derechos humanos (tanto universal como regional), así como la falta de información sobre las diferentes instancias de los mecanismos de protección y sus seguimientos. Se percibe además que no existe un señalamiento sobre los instrumentos de Derecho Internacional de los Derechos Humanos (DIDH) ratificados por el Estado uruguayo, excepto la Declaración Universal de Derechos Humanos y la Convención de los Derechos del Niño.

También podemos ver en el programa un tratamiento desparejo de elementos conceptuales sobre la concepción del sujeto integral y de educación integral. Si bien existe una tendencia hacia la integralidad, sobre todo discursiva, esto no se refleja en la formulación y tratamiento que se da al interior de cada área de conocimiento. Se encuentran contenidos esquematizados, listado de contenidos e información a ser trabajada, lo que da un resultado algo atomizado. Esto no facilita el tratamiento de estos contenidos en la práctica de forma integral e interrelacionada.

La forma en que los contenidos y temáticas son presentados no estimulan la integración y transversalización propuesta en los objetivos del programa. Se plantean algunas dudas respecto a su implementación. Por ejemplo ¿está todo el cuerpo docente capacitado para llevar adelante este Programa con una concepción acorde con la $\mathrm{EDH}$ ? ¿O en la práctica queda librado a la comprensión y sistematización que cada docente realice para llevarlo adelante, según una visión de EDH y educación participativa, o como una mera reproducción de los contenidos?

\section{Especificidades del trabajo en campo}

Esta fase de la investigación fue la más desafiante, en la medida que propuso la aplicación de nuevas técnicas y el adaptarse a los tiempos y formas de funcionamiento instituciocnales.

Para el desarrollo de esta fase de investigación fue importante diseñar una estrategia en diferentes etapas (ingreso a los centros, estrategia de intervención y estrategia de cierre). 
Hubo que organizar, y por momentos reorganizar, los datos de forma que pudieran dar cuenta de un proceso de producción de conocimiento. En varios momentos tuve que replantear las estrategias de acercamiento al objetivo de investigación, readecuando las modalidades de acceso.

En un primer momento había planificado como estrategia de intervención en las escuelas solicitar observar una clase por grado para cubrir los seis niveles que abarcan el sector de primaria. Pero luego de la estrategia de ingreso a los centros educativos que implicaba conseguir los permisos formales de las autoridades, así como alcanzar la disposición y acercamiento necesario de las inspectoras, directores y equipos docentes a través de una presentación personal y una presentación del trabajo de tesis a desarrollar, tuve que cambiar la modalidad de intervención por percibir actitudes de resistencia en el contacto espontáneo.

Alcanzar la confianza necesaria para lograr los climas intrasubjetivos apropiados para esta investigación fue fundamental. Ser paciente con los docentes, escucharlos primero, ser una presencia frecuente, conversar y esperar que ellos fueran quienes me invitaran a sus aulas u otras actividades, terminó siendo la estrategia emprendida siempre con el objetivo de establecer vínculos de confianza, de respeto hacia ellos y su labor profesional.

Fue ya transcurrido un mes, aproximadamente, de mi presencia en las escuelas, con una concurrencia semanal durante la cual me entrevistaba con los directores, secretarias, maestras comunitarias o integradora, interactuaba en los recreos con los maestros y maestras, así como con los niños y niñas, que fui accediendo a ser invitada a las clases o a presenciar algunas de las tareas significativas para los equipos docentes, como ser los talleres, los grupos integrados donde trabajan varios docentes.

La estrategia de cierre llevada a cabo en el trabajo de campo consistió en un taller con el equipo de maestros y maestras de cada una de las escuelas al finalizar el trabajo. Esta instancia consistió en una primera parte, trabajar en grupo con los y las docentes sobre sus opiniones sobre la EDH. También se destinó 
en esta instancia de cierre, a trabajar en la devolución y algunas conclusiones de la investigación, ya que los maestros y maestras fueron actores de la misma, y por lo tanto es importante realizar un cierre temático y afectivo por la disposición, la información y la confianza brindada.

\section{Conclusiones}

La incorporación de la EDH en el sistema educativo uruguayo implicó una adecuación a varios niveles. El ajuste de la normativa educativa a las tendencias internacionales de $\mathrm{EDH}$, que el Uruguay está asumiendo, lleva a trascender la dimensión declarativa para enfocar su despliegue a nivel curricular y buscar la concreción en las prácticas educativas cotidianas.

La investigación constató la existencia de un ámbito educativo heterogéneo donde coexisten diferentes concepciones educativas y varios imaginarios del rol docente (la instrucción, la capacitación, el rescate social y la formación de sujetos de derecho) que dan lugar a una expresión conflictiva, desafiante y de transformaciones en las prácticas educativas. Por eso la investigación se movió en varios niveles (ley de educación, programa de estudios y prácticas escolares), buscando examinar diferentes dimensiones y su relación mutua, a fin de lograr una comprensión compleja del ámbito educativo y aportar a la incorporación de la EDH a la educación pública uruguaya.

La investigación realizada permite extraer las siguientes conclusiones:

\section{Existen avances importantes en la Ley General de Educación $N^{\circ} 18.437$ de 2008 en cuanto a la incorporación de la EDH en el sistema educativo}

La ley es un paso decisivo en la incorporación de la EDH en el Uruguay al plantear como punto de partida a la educación como un derecho humano. La formulación de ejes transversales que destaca a la EDH como derecho en sí misma y parte importante del derecho a la educación (artículo 40.1), implica 
un avance en política educativa. Con esta misma orientación creó un ámbito específico para el desarrollo de este enfoque: la Comisión de EDH en calidad de asesora del Sistema Nacional de Educación Pública (artículo 110).

\section{La actualización del Programa de educación inicial y} primaria en el ámbito de la Administración Nacional de Educación Pública (ANEP, 2009) incluye progresos visibles pero plantea contradicciones para la implementación de la EDH

En la introducción del nombrado documento, los derechos humanos son una idea central. Están desarrollados ampliamente en su justificación y en la creación de un área de conocimiento ciudadano desplegada en las disciplinas de Ética y Derecho. Se incorporan de esta manera contenidos específicos y un planteo de la tarea educativa que destaca la participación activa de las y los educandos en los procesos integrales de enseñanza y aprendizaje y explicita su condición de sujetos de la educación y sujetos de derecho. No obstante, a pesar de los elementos que representan un avance importante en la incorporación de EDH en el Programa, el documento contiene algunos puntos que generan debilidades en el abordaje de la $\mathrm{EDH}$ en las prácticas. Ellos son la desagregación en disciplinas separadas y los listados de contenidos específicos, que no favorecen el desarrollo de un enfoque integral y transversal de la EDH.

\section{La puesta en práctica de la $\mathrm{EDH}$ en la labor educativa concreta está atravesada por una serie de dificultades que obstruyen su implementación}

\subsection{Falta de formación docente}

Existe un desconocimiento de los derechos humanos y de la EDH de parte de los y las maestras, en cuanto a sus contenidos, elementos valóricos y orientación para las prácticas escolares. Lo anterior genera, por un lado, un abordaje parcializado y por momento descontextualizado de la práctica educativa integral y, por otro lado, la dificultad de identificar los elementos 
pedagógicos, académicos, valóricos y actitudinales que forman parte de la $\mathrm{EDH}$.

El trabajo de campo permitió comprobar que distintos contenidos del Programa relacionados con la EDH y los derechos humanos no siempre eran comprendidos y abordados por los y las docentes como tales. Tal incomprensión obstruye el alcance de los objetivos de la propuesta y parcializa o diluye los contenidos de derechos humanos.

En gran parte del cuerpo docente se aprecia falta de conocimientos sobre el enfoque de la EDH como abordaje pedagógico y como una estrategia integral y transversal de la tarea educativa, encuadrada en fines y objetivos específicos. Como contraste, al mismo tiempo se identificó que muchos docentes están trabajando con metodologías y abordajes muy afines al enfoque de $\mathrm{EDH}$, pero no lo identifican como tal. Esto parece deberse a que no cuentan con una buena comprensión de qué son los derechos humanos y cómo se concretan en la vida cotidiana de la convivencia.

\subsection{Falta de recursos, técnicos y formativos que permita a los y las docentes instrumentar la EDH desde un abordaje integral y transversal}

Los y las docentes son conscientes de sus desconocimientos o parte de ellos y plantean que necesitan actualizarse con una formación en EDH. Para incorporar este enfoque rescatan y valorizan los espacios de trabajo en equipo y de construcción dentro de los centros que encaren las problemáticas que se dan en las escuelas. Los equipos docentes reclaman mayor espacio para la reflexión y la problematización de la tarea educativa en la actualidad, como necesidad de co-construir visiones compartidas y abordajes conjuntos en las escuelas.

Como consecuencia del desconocimiento a menudo se reduce la EDH a actividades puntuales, a comentarios sobre contenidos específicos con poca contextualización, y a la incapacidad 
para identificar algunos contenidos del propio Programa como temas de EDH.

La participación de los niños y niñas dentro de las escuelas está mal abordada, lo que produce una ausencia u omisión de esta dimensión del trabajo educativo tan importante para la $\mathrm{EDH}$.

3.3. Falta de valorización de la temática en la evaluación y en la planificación estratégica

La mayoría de los y las docentes no jerarquizan la EDH en sus planificaciones, ni en su proceder, sea este académico (planificado) o espontáneo (de relación y comunicación diaria). Reconocen que no poseen recursos claros para la evaluación y monitoreo de espacios de su trabajo que involucren la $\mathrm{EDH}$, desde elementos cognitivos hasta habilidades valóricas, éticas y para la acción. Existe la percepción en los maestros y maestras que el cuerpo inspectivo no valora, o no sabe como implementar estas áreas de trabajo, razón por la cual no sienten que se los guíe y capacite en la materia.

\section{Incomunicación entre las autoridades, los especialistas encargados de desarrollar la política educativa (el pensar y escribir) y el cuerpo docente responsable de desarrollar la tarea educativa propiamente dicha (el hacer)}

Se percibió claramente en diferentes oportunidades que existían problemas de incomunicación y falta de comprensión entre estos diferentes niveles y actores del sistema educativo. En consecuencia aparecen sentimientos de malestar docente ante las autoridades y las solicitudes de la burocracia escolar y acciones desarticuladas en la asignación de recursos técnicos o económicos y en las respuestas a las necesidades de las escuelas. El magisterio manifiesta sentirse desvalorizado por partes de las autoridades y los agentes de planificación y programación educativa. 


\section{Se identificaron prácticas educativas que no son visualizadas como EDH pero que deberían ser tomadas en cuenta para una real incorporación de la EDH en el sistema educativo. Deberían ser consideradas como puntos de partida para afianzar y desarrollar la EDH en el trabajo que los docentes ya están desarrollando}

Si bien se identificaron debilidades del enfoque de EDH en las prácticas educativas, también se encontraron elementos alentadores para la implementación de un enfoque de EDH. Se trata de búsquedas de metodologías acordes a la educación integral y que promueven la convivencia desde el respeto y desarrollo de las personas.

Se observó en las tres escuelas estudiadas una labor de los equipos docentes con tendencia a desarrollar actividades planificadas, articuladas y coordinadas con un enfoque integral y participativo de la tarea educativa. Eso marca una tendencia positiva y esperanzadora, pues sugiere que los y las maestras están buscando transformar sus prácticas con una orientación hacia la $\mathrm{EDH}$, aunque con frecuencia no lo expresen en estos términos.

En las escuelas se destaca en general un trabajo intenso y comprometido hacia las tareas educativas. Aunque esto no necesariamente salga a la luz más allá del contexto concreto de cada escuela, se aprecia una preocupación por la vida escolar al interior de los centros, por procurar que cada día, cada momento que transcurre en la escuela, sea armónico y amigable entre los diferentes actores de la comunidad educativa.

En síntesis, si bien el ámbito educativo está marcado por múltiples tensiones y situaciones conflictivas, en las que aparecen diferentes concepciones sobre la tarea educativa por desarrollar y diferentes compresiones y abordajes docentes -lo cual constituye un escenario heterogéneo y difícil para instalar la EDH - existen elementos alentadores y auspiciosos que pueden ser puntos de apoyo para profundizar y consolidar un enfoque sólido de EDH. 


\section{Bibliografía utilizada}

Congreso del Uruguay (2008). Ley General de Educación $N^{o} 18.437$. Montevideo, Uruguay.

Magendzo, Abraham (2008). La escuela y los derechos bumanos. Ediciones Cal y Arena, México.

Morin, Edgar: (1998). Articular los saberes, ¿Qué saberes enseñan en las escuelas?. Ediciones Universidad del Salvador, BsAs, Argentina.

Organización Naciones Unidas (2005). "Plan de acción para la primera etapa (2005-2007) del Programa Mundial para la educación en derechos humanos". Aprobado por la Asamblea General el 2 de marzo de 2005.

Organización de Estados Americanos (1989) Protocolo adicional a la Convención Americana sobre derechos humanos en materia de derechos económicos, sociales y culturales. (OEA, 1989) Protocolo de San Salvador.

Rodino, Ana María. (1999) "La educación en valores entendida como educación en derechos humanos. Sus desafíos contemporáneos en América Latina." Publicado en la Revista IIDH/ Instituto Interamericano de Derechos Humanos, $\mathrm{N}^{\circ} 29$, enerojunio 1999, San José, Costa Rica.

- (2003). "Educación para la vida en democracia: Contenidos y orientaciones metodológicas". Cuadernos Pedagógicos, IIDH.

Tarres, María Luisa, (2001) "Observar, Escuchar y comprender, sobre la tradición cualitativa en la investigación social", Presentación, Prólogo, Cap:"Lo cualitativo como tradición". Editado por Facultad Latinoamericana de Ciencia Sociales, El Colegio de México. 